\title{
FUNDAMENTOS TEÓRICOS Y CRÍTICOS DE LA ESCRITURA AUTOBIOGRÁFICA FEMENINA EN LENGUA FRANCESA
}

\author{
THEORETICAL AND CRITICAL FUNDAMENTALS OF WOMEN'S \\ AUTOBIOGRAPHICAL WRITING IN THE FRENCH LANGUAGE
}

\author{
Ángela Magdalena ROMERA PINTOR \\ Universidad Nacional de Educación a Distancia \\ aromera@flog.uned.es
}

\begin{abstract}
Resumen: El propósito de este estudio es analizar a grandes rasgos el concepto de género, la evolución y la tipología de textos de la escritura autobiográfica, así como la producción autobiográfica en lengua francesa de autoría femenina, con objeto de ofrecer una fundamentación teórica y crítica actualizada como marco de referencia para las distintas aportaciones que componen el presente monográfico, centrado en los siglos XIX, XX y XXI.
\end{abstract}

Palabras clave: Escritura autobiográfica. Escritura femenina en lengua francesa.

Abstract: This study aims to broadly analyse the concept of genre, the evolution and the texts' typology of autobiographical writing, as well as the production in French of feminine authorship, in order to establish the most up-to-date research in the matter and to offer theoretical and critical fundamentals as a reference framework for the contributions contained in this monograph, which focuses on the $19^{\text {th }}, 20^{\text {th }}$ and $21^{\text {st }}$ centuries. 
Key Words: Autobiographical writing. Women's writing in French.

\section{INTRODUCCIÓN}

Se publica esta sección monográfica en la revista Signa, dentro de las actividades del Centro de Investigación de Semiótica Literaria, Teatral y Nuevas Tecnologías (SELITEN@T), dirigido por el profesor José Romera Castillo, siendo la escritura autobiográfica una de sus líneas de investigación pioneras y más productivas en España. Ejemplo de ello son las diversas actividades que ha dedicado al tema, tal y como se pone de manifiesto en el trabajo de Romera Castillo (2010), "La escritura (auto) biográfica y el SELITEN@T: Guía bibliográfica”. En particular, el Centro ha consagrado cuatro Seminarios internacionales a este campo de estudio, seminarios que, a su vez, han dado lugar a cuatro volúmenes ${ }^{1}$, en los que se recogen algunas contribuciones específicamente relacionadas con la escritura autobiográfica en lengua francesa. Asimismo, ha sacado a la luz numerosas publicaciones, de entre las que también destacaré, por su relevancia en la materia que nos ocupa, la de Romera Castillo (2008), De primera mano. Sobre escritura autobiográfica en España (siglo XX), libro que dedica uno de sus capítulos a los textos autobiográficos traducidos al español en estos últimos años.

Por lo demás, el campo de estudio del presente monográfico, dentro del ámbito lingüístico francófono, pretende abarcar tanto las memorias, como los escritos propiamente autobiográficos, así como la autoficción, razón por la que hemos elegido la expresión genérica "escritura autobiográfica" que se recoge en el título. En este sentido, hemos optado por considerar la noción de "escritura autobiográfica" en su acepción más amplia, en la medida en que permite tener en cuenta una mayor variedad de textos. Recordemos, como hace observar Marcel de Grève², que el término

1. Las actas de estos cuatro Seminarios internacionales, centrados en los diversos géneros, han sido editadas por José Romera Castillo et alii. Se trata de los siguientes volúmenes: Escritura autobiográfica (1993), Biografias literarias (1975-1997) (1998), Poesía histórica y (auto)biográfica (1975-1999) (2000), así como Teatro y memoria en la segunda mitad del siglo XX(2003).

2. "Le terme autobiographique peut prendre un sens strict de récit rétrospectif de la vie d'un écrivain [...], rédigé par lui-même. [...] Dans ce sens, l'autobiographie se distingue nettement des mémoires qui mettent l'accent sur les événements extérieurs ou sur la réflexion politique ou philosophique, ainsi que du journal intime, rédigé au jour le jour. [...] Mais l'autobiographie peut aussi devenir une notion très étendue. Le terme peut désigner tout écrit, où l'auteur (écrivain ou non) rend compte de sa propre vie. Selon aussi bien les Anglaises 
"autobiográfico" recoge esta doble acepción, una en sentido estricto, la otra en sentido amplio.

Una vez señaladas estas premisas, se hace necesario establecer los fundamentos teóricos y críticos en la materia. Para ello, y antes de adentrarnos de manera específica en la producción de autoría femenina, nos ocuparemos en un primer momento de las principales argumentaciones que sustentan el debate en torno al género, así como a las definiciones y tipologías de las principales formas autobiográficas que serán objeto de estudio en los artículos que componen este monográfico.

\section{EL GÉNERO AUTOBIOGRÁFICO}

Como es sabido, el debate en torno al género autobiográfico se desarrolló principalmente en los años 70 del siglo pasado. El propio Philippe Lejeune señalaba unos presupestos de permanencia y autonomía sobre los que se fundamentaría la clasificación del género: "Le genre [...] implique donc la croyance en une espèce d'identité, qui ne peut être produite que par des séries de distinctions et de préceptes destinés à la fois à isoler le genre des autres productions, et à hiérarchiser et centrer le domaine ainsi enclos" (1975b: 911). En su estudio "Autobiographie et histoire littéraire", Lejeune aborda desde una perspectiva crítica distintas aportaciones en relación al género autobiográfico, como las de Élisabeth E. Bruss, Francis R. Hart o William L. Howard ${ }^{3}$. Pero será sin duda la definición del "pacto autobiográfico"4 de Lejeune la que se convertiría pronto en el centro de la polémica. De ello da buena cuenta el artículo de Martine Renouprez, 'L'Autobiographie en question: poétique d'un

Christina Ruse et Marilyn Hepton [...] que, par exemple, les Françaises Joëlle Gardes-Tamine et Marie-Claude Hubert [...], le genre autobiographique peut, au sens large, comprendre, outre le type de récit évoqué ci-dessus, des mémoires, des récits de souvenirs, des journaux intimes, des lettres, voire des romans inspirés de la vie de l'auteur" (Grève, 2008: 23-24).

3. Lejeune cuestiona las aportaciones de Hart (en "Notes for an Anatomy of Modern Autobiography") y de Howard (en "Some principles of Autobiography") y concluye destacando en ellos la ambigüedad de su planteamiento: "Les analyses de Hart et de Howarth se trouvent donc, par rapport à la critique du genre, dans une position ambigüe: d'un côté, elles participent à la mythologie du genre, [...] de l'autre, elles contestent cette mythologie en refusant les jugements de valeur, en rétablissant une pluralité de modèles, et en pratiquant une analyse dissociative des facteurs" (Lejeune, 1975b: 917).

4. Según Lejeune, el pacto autobiográfico consiste en "l'affirmation dans le texte de cette identité [celle du nom (auteur-narrateur-personnage)], renvoyant en dernier ressort au nom de l'auteur sur la couverture. Les formes du pacte autobiographique sont très diverses: mais, toutes, elles manifestent l'intention d'honorer sa signature. Le lecteur pourra chicaner sur la ressemblance, mais jamais sur l'identité" (Lejeune, 1975a: 26). 
genre", en donde se aborda la evolución del concepto de autobiografía $\mathrm{y}$, en particular, las argumentaciones de algunos críticos que rebaten la definición restrictiva ideada por Lejeune, tales como Bruss y Gisèle Mathieu-Castellani.

En las más recientes revisiones sobre el concepto de género autobiográfico, se ha llegado a concluir que se debería considerar más bien como la manifestación de una variedad de formas. Así lo plantea Pierre Brunel en su artículo "Introduction aux autobiographies" (2008: 14). Según esta concepción de la escritura autobiográfica, sólo habría dos polos extremos que establecerían los límites:

Deux limites devraient pouvoir être fixés: la fiction pure, à laquelle ne viendrait se mêler aucun élément suspect de procéder d'une autobiographie déguisée de l'auteur; l'autobiographie pure, qui s'interdirait de faire place à des détails inventés. Entre les deux évolue la masse même de la littérature, et pas seulement de la littérature romanesque (Brunel, 2008: 10).

En definitiva, Brunel llega a proponer que la autobiografía se considere un modo de relato, más que un "género" o "sub-género" propiamente dicho: "Plutôt que de parler de genre, ou de 'sous-genre', je considérerai l'autobiographie comme mode de récit" (2008: 15).

La vigencia del interés por dilucidar la cuestión en torno al género autobiográfico se pone de manifiesto en estos estudios que siguen abordando la cuestión en nuestros días, tal y como hace también Marcel de Grève en su artículo "L'autobiographie, genre littéraire?", donde empieza por calificarlo de "género problemático". Además de rastrear los textos autobiográficos con anterioridad y posterioridad a Rousseau, Grève destaca la presencia de un conflicto que viene a constituir una característica propia de la autobiografía: "Le genre autobiographique [...] est exposé à deux écueils principaux et souvent complémentaires: soit une complaisance narrative incontrôlée, soit un étalage présomptueux et volontiers narcissique des sentiments" (2008: 25). 


\section{MEMORIAS, AUTOBIOGRAFÍA Y AUTOFICCIÓN}

Junto al debate en torno al género autobiográfico e indisolublemente ligado a éste, se ha tratado de ofrecer asimismo la definición de los distintos tipos de textos en los que se manifiesta la escritura autobiográfica, labor que, a menudo, también se ha abordado de forma paralela al estudio de la evolución de los mismos en el tiempo.

Sin necesidad de remontarse a las Confesiones de san Agustín, consideradas como una de las primeras manifestaciones de la escritura autobiográfica, la mayoría de los críticos coinciden en tomar a Rousseau como referente y precursor de la autobiografía en sentido propio, sobre todo en el ámbito francófono ${ }^{5}$. Es lo que Gusdorf destaca en los siguientes términos:

L'intervention de Rousseau est décisive parce que, d'une part, il est animé par les intentions profondes propres aux auteurs d'autobiographies; il éprouve le besoin de ressembler sa vie et de la soumettre au jugement de Dieu et des hommes. Mais d'autre part, et en même temps, il mène à bien le chef-d'ouvre qui parachève une exceptionnelle carrière d'artiste de la prose. La réussite commerciale fera le reste. [...] L'autobiographie littéraire possède désormais un modèle qui fixe un contenu, impose un projet et un ton, des thèmes obligés (Gusdorf, 1975: 965).

Al establecer las Confesiones de Rousseau como punto de inflexión del concepto de autobiografía en sensu stricto, cobran importancia las teorías ${ }^{6}$ y los razonamientos que se han esgrimido a la hora de ofrecer

5. En el ámbito francófono, cuatro publicaciones recientes se sitúan como obras de referencia en el campo de la autobiografía. Se trata de los cuatro volúmenes editados por el Servicio de Publicaciones de la Universidad de Cádiz, que se ocupan de la autobiografía en Bélgica, África, el Maghreb, así como el Caribe y el Océano índico. También se ocupa de la autobiografía en el ámbito francófono, en particular del Maghreb, el artículo de Anne-Marie Gans-Guinoune "Autobiographie et francophonie: cache-cache etre "nous' et 'je"”. Por su parte, Susanne Gehrmann y Claudia Gronemann han editado en 2006 un monográfico centrado en el tema: Les enJEux de l'autobiographique dans les littératures de langue française: du genre à l'espace, l'autobiographie postcoloniale, l'hybridité.

6. En este contexto, además de Lejeune y de Jean Starobinski, abordan el tema los estudios de Georges Gusdorf, Damien Zanone, Yves Coirault, así como más recientemente Jacques Lecarme y Éliane Lecarme-Tabone, y Jean-Louis Jeannelle. En relación a las aportaciones de Georges Gusdorf en la materia, resulta de interés el muy completo artículo de Jesús Camarero "La théorie de l'autobiographie de Georges 
una clara distinción entre memorias y autobiografía. Ésta se centraría en el relato en primera persona de los acontecimientos ligados a la vida íntima y personal del autor, a su carácter y personalidad, al tiempo que las memorias se ocuparían, en cambio, de relatar los hechos históricos, sociales o políticos, con una clara voluntad testimonial.

La definición de autobiografía propuesta por Lejeune ${ }^{7}$ se ha tomado a menudo como referencia en las revisiones posteriores del concepto. Y en este contexto, a la hora de definir la frontera que separa la autobiografía de las memorias, se ha cuestionado una vez más su teoría del "pacto autobiográfico". No sólo recientemente Lecarme, sino ya en su momento, Bruss o Yves Coirault expresaron la dificultad de establecer una división categórica y exclusivista de ambos tipos de texto. En su artículo intitulado "L'autobiographie considérée comme acte littéraire", Bruss cuestionó el "pacto autobiográfico" por cuanto le atribuyó un desfase cronológico y, por lo mismo, conceptual, entre las partes del contrato. Retomando este planteamiento de Bruss, Yves Coirault argumentó en torno al "narcisismo" del autor como referente común de los escritos autobiográficos:

Qu'il s'agisse de "Mémoires", de "Souvenirs", d'"Autobiographies" [...], l'acte autobiographique commence, ou recommence, par une inversion de la fonction spéculaire qui caractérise la littérature de témoignage: l'auteur tourne alors son miroir vers lui-même, "passé présent”; il se regarde vivre, avoir vécu. Lorsque le mémorialiste s'appelle Retz, Saint-Simon, Chateaubriand, l'Histoire est là; mais aussi Narcisse historien, lié à ses souvenirs d'existence par la vertu de la mémoire et selon un pacte plus ou moins secret (Coirault, 1975: 950).

De esta manera, Coirault concluye con un enfoque menos excluyente que Lejeune, limitándose a destacar como marca distintiva de la autobiografía su carácter narcisista, aquello que denomina la

Gusdorf" (2008: 57-82), en donde expone la teoría y los estudios de Gusdorf sobre la escritura del yo y la escritura autobiográfica. El monográfico editado en 1993 por José Romera Castillo y Alicia Yllera, entre otros, bajo el título Escritura autobiográfica, recoge algunos artículos en el ámbito francófono a pesar de estar centrado principalmente en el campo de la autobiografía hispánica.

7. "Récit rétrospectif en prose qu'une personne réelle fait de sa propre existence, lorsqu'elle met l'accent sur sa vie individuelle, en particulier sur l'histoire de sa personnalité" (Lejeune, 1975a: 14). 
“complacencia de uno mismo", la "autofascinación":

La marque de l'Autobiographie, celle que je crois retrouver dans des ouvres d'époques diverses, demeure la complaisance à soi, et, dans le pire ou le meilleur des cas, l'autofascination [...]. Qu'elle ait évolué dans le sens d'une littérature plus intime, on ne saurait s'en étonner (Coirault, 1975: 950-951).

También Gusdorf se esforzó por demostrar la imposibilidad de deslindar autobiografía y memorias. Su principal tesis de trabajo reside en su convicción de que ambos tipos de texto no sólo no se oponen entre sí, sino que confluyen de forma concéntrica. En otros términos, insiste en la imposibilidad de deslindar lo privado de lo público en la vida del autor, razón por la que la distinción basada en el carácter privado o público del escrito resulta, a su entender, insuficiente para clasificar el texto como autobiografía o como memorias:

Ainsi serait manifestée l'insuffisance et l'arbitraire de l'opposition entre Autobiographie et Mémoires, assimilée à la distinction entre l'ordre privé et l'ordre public dans une vie personnelle, comme s'il était possible d'opérer une telle dissociation. [...] Autrement dit, mémoires et autobiographies ne seraient pas opposées, mais plutôt concentriques, selon l'importance respective reconnue par le narrateur à la vie privée et à la vie publique, sans que l'une puisse tout à fait éliminer l'autre (Gusdorf, 1991: 269-71).

Así, memorias y autobiografía entrarían a formar parte de un concepto más amplio, al que denomina la escritura sobre uno mismo: "Le plus simple, le plus prudent est d'homologuer comme écriture du moi tout texte rédigé à la première personne où l'auteur porte témoignage de sa propre vie" (Gusdorf, 1991: 57). Considerando imposible de establecer una frontera entre memorias y autobiografía, Gusdorf incide en el interés histórico o meta-histórico del autor, para identificar la autobiografía con una experiencia iniciática: 
Toute autobiographie digne de ce nom présente ce caractère d'une expérience initiaque, d'une recherche $d u$ centre. L'intérêt porté au récit des épisodes successifs, le pittoresque des anecdotes ne doit pas faire illusion. À la différence des Mémoires, de caractère narratif, dont l'intérêt est essentiellement historique, l'autobiographie est animée par une intention méta-historique; elle se situe selon l'ordre d'une ontologie de la vie personnelle (Gusdorf, 1975: 971).

Lecarme y Lecarme-Tabone inciden en la ejemplaridad a la hora de establecer la distinción entre autobiografía y memorias, así como en el objeto de la escritura, siendo el de la autobiografía el de la totalidad del individuo, tanto público como privado:

Disons qu'en général l'autobiographie vise dans le sujet vivant l'individu, mais tout l'individu, avec sa vie privée et publique. [...] Les mémoires supposent, au contraire, une sectorisation de l'individu [...], le mémorialiste fonctionne sur l'illusion de l'exemplarité [...]. Les mémoires impliquent toujours une importance sociale qui peut être liée à des fonctions, à des événements, à des positions dominantes (1999: 49-50).

Por su parte, Damien Zanone se propuso demostrar la dificultad de encasillar las memorias como un género estable. Analiza las razones que llevaron al fracaso de las memorias tras el boom que experimentaron en las primeras décadas del siglo XIX y llega a concluir que el abandono de esta modalidad de escritura se fundamenta en la dificultad insuperable que conlleva la memoria histórica, aquello que denomina "l'embarras poétique" (1990: 45). Se trata, a su juicio, del conflicto que se plantea entre el yo testimonial y la memoria histórica: "l'effacement de soi dans le récit de sa mémoire historique est tenu pour moralement nécessaire, mais poétiquement impossible" (1990: 47). Zanone atribuye el origen de la crisis de las memorias a la emergencia del individualismo como valor moral y político desde finales del XVIII: "l'expression de soi est saisie comme un besoin moral [...]. Cela met la tradition des Mémoires en difficulté: [...] ce 
genre aristocratique doit se réinventer" (1990: 48).

La "reinvención" de las memorias permitirá, por lo demás, su supervivencia. Esta evolución y pervivencia de las memorias en pleno siglo XX, a pesar de la crisis subvenida tras la profusión de textos de los memorialistas en la primera mitad del XIX, se aborda en el libro de JeanLouis Jeannelle ${ }^{8}$ Écrire ses mémoires au $X X^{e}$ siècle. Déclin et renouveau. $\mathrm{Su}$ punto de partida es la definición de Furetière, según la cual las memorias se consideran libros de historiadores que han tenido parte o han sido testigos de los acontecimientos (2008: 14). Distingue tres períodos que comprenden la III ${ }^{\mathrm{a}}$ República, la Segunda guerra mundial y el último tercio del siglo XX. A medida que la historia "irrumpe en la vida de cada francés", las memorias comienzan a ejercer un papel de "reconfiguración del pasado histórico" (2008: 16-17).

[...] les Mémoires attestent une vie dans sa dimension publique et collective: [...] regard porté sur une période historique circonscrite (guerre, crise nationale, génération), peinture d'une action, politique, militante ou professionnelle, ayant conduit son auteur à se tenir au cour des conflits d'une époque donnée. Les Vies majuscules constituent bien au XX' siècle un genre à part entière; elles représentent à la fois un modèle narratif où la mémoire peut prétendre exercer une ambition historiographique et un archétype littéraire qui connaît une évolution distincte de l'autobiographie, mais d'égale importance; celui des récits égohistoriques (Jeannelle, 2008: 13).

Para Sébastien Hubier la crisis de fantasía que sufre la novela hacia finales del XIX se convertirá en el punto de partida de la nueva novela autobiográfica. Se produce así la paradoja de querer presentar una ficción que sea real y que al mismo tiempo se reconozca como una invención:

8. Más recientemente, en 2013, Jeannelle y Zanone, junto con Marc Hersant, han dirigido otra publicación que trata sobre la renovación del concepto de memorias, intitulada Le Sens du passé. Pour une nouvelle approche des Mémoires. Por su parte, Zanone ha publicado en 2006 su tesis, revisada y actualizada, bajo el título Écrire son temps. Les Mémoires en France de 1815 à 1848, donde analiza el género a través de un corpus de once textos. 
Il s'agit alors de créer un nouveau jeu du réel et de l'imaginaire, dans le cadre du genre romanesque auquel ils ouvrent une possibilité de renouvellement-par exemple en conférant à un récit autobiographique l'apparence d'une fiction dans laquelle l'écrivain se sentirait plus libre de s'exprimer (Hubier, 2005: 111).

La evolución de la autobiografía hacia la autoficción ${ }^{9}$, que caracteriza buena parte de la producción del siglo XX hasta nuestros días, ha sido abordada por Thomas Régnier en su artículo "De l'autobiographie à l'autofiction: une généalogie paradoxale". También aquí se erige a Rousseau como precursor, aunque esta vez no tanto por sus Confesiones, cuanto más bien por su obra Rêveries du promeneur solitaire, donde se expresa la reivindicación de una verdad subjetiva: "une sincérité préférée à la froide vérité factuelle" (2008: 34). A partir de ahí, Régnier hace un balance de las razones que fundamentan la preferencia por la ficción en la literatura contemporánea. Desarrolla el tema a través de las consideraciones de Jean Starobinski y termina por destacar la seducción que llega a ejercer la ficción frente a la fidelidad a la verdad:

Force est de constater aujourd'hui, sur le plan d'une axiologie littéraire, la supériorité de la fiction sur le compte rendu factuel. [...] Il y a dans l'autofiction l'idée que l'être ne pourra atteindre sa vérité tant qu'il ne se sera pas élargi, mis en question par la fiction. On mesure ici la séduction de l'autofiction, comme les risques qu'elle comporte (Régnier, 2008: 35-36).

Establecidas, así, las ventajas de la autoficción frente a la autobiografía, Régnier no deja de llamar la atención también sobre los riesgos ${ }^{10}$ que conlleva: de un lado, la disolución de lo real en la ficción; de

9. Como se sabe, el término "autoficción" fue acuñado por Serge Doubrovsky para definir su novela Fils. En relación al tema de la autoficción, son numerosos los críticos que le han dedicado su atención, tales como, entre otros muchos, Manuel Alberca, Ana Casas, Éric Chevillard, Vincent Colonna, Marie Darrieussecq, Laurent Jenny o Jacques Lecarme.

10. En este contexto, Marcel de Grève hace observar la dificultad de distinguir en ocasiones la autobiografía de la novela de ficción en primera persona: "S'il est vrai que l'autobiographie ne ressortit pas, à proprement parler, à la littérature de fiction, puisque l'auteur y apparaît à la fois comme narrateur et comme personnage principal, et qu'il est censé reproduire la vérité, dans la pratique il n'est pas toujours aisé de 
otro, el recurso a la ficción como mero subterfugio para confesar simple y llanamente lo íntimo: "L'écueil du tout-fictionnel: où le réel, au lieu d'être affronté, se dilue dans la fiction. L'écueil du tout-autobiographique: où la fiction sert d'alibi au déballage pur et simple de l'intime" (2008: 36).

El poder evocador de la autoficción frente a la autobiografía se destaca también en el estudio "L'étiquette générique autofiction: us et coutumes". Aquí, Mar García subraya la dimensión lúdica de la autoficción, que invita al lector a participar de forma creativa: "une invitation à lire entre lignes, à dévoiler des énigmes, en un mot, à participer à la lecture de manière créative" (García, 2009: 150). Aún cuando el debate teórico y genérico en torno la autoficción no se ha cerrado, García concluye subrayando su vinculación con la Postmodernidad: "la Postmodernité, et les valeurs esthétiques qu'elle véhicule, est inséparable de la prédisposition autofictionnelle de la littérature actuelle" (2009: 160).

\section{ESCRITURA AUTOBIOGRÁFICA FEMENINA}

De entrada, importa recordar aquí el debate en torno a la existencia de una especificidad en la labor escritural llevada a cabo por mujeres. Especialistas en la materia, como Béatrice Didier (L'écriture-femme), Christine Planté (La petite sœur de Balzac: essai sur la femme auteur), o Martine Reid (Des femmes en littérature), entre otros ${ }^{11}$, se han ocupado de esta cuestión. Más recientemente, Delphine Naudier ha abordado de nuevo el tema en su artículo "L'écriture-femme, une innovation esthétique emblématique", donde analiza el resultado del debate originado por los postulados de Simone de Beauvoir, Annie Leclerc o el controvertido concepto de "escritura femenina", acuñado por Hélène Cixous ${ }^{12}$. En particular, Naudier destaca la manifestación -en los años

distinguer l'autobiographie de certains romans écrits à la première personne; il convient, en fait, de déterminer dans quelle mesure l'auteur s'identifie au personnage, ce qui ne peut se faire qu'à l'aide de confidences de l'auteur lui-même ou à partir de données se trouvant dans le même texte" (2008: 24).

11. Jeannelle también ha tratado el tema en "Le sexe des Mémoires", que se incluye en los estudios recogidos por Anne Coudreuse en Les Mémoires, une question de genre?, monográfico donde se aborda la controvertida cuestión de la especificidad de la escritura autobiográfica femenina. Por su parte, Josefina Bueno Alonso aborda la cuestión en el contexto del Maghreb francófono en su artículo "Femme, identité, écriture dans les textes francophones du Maghreb".

12. Sobre la relevancia del pensamiento y la obra de Cixous remitimos a Marta Segarra, que ha publicado en 2010 Entrevistas a Hélène Cixous. No escribimos sin cuerpo. La obra reúne 12 entrevistas en las que Cixous reflexiona, entre otros temas, sobre écriture féminine, "que constituye una de sus aportaciones teóricas más célebres y polémicas al pensamiento feminista”, según se recoge en la Nota de la editora en la 
70 - de una singularidad literaria que glorifica lo femenino, y, por lo mismo, todo aquello que directamente remite al cuerpo de la mujer, como pudieran ser las experiencias sexuales, el embarazo o la menstruación:

Le "féminin" en écriture va dès lors être un moyen pour une partie des auteurs féminins majoritairement universitaires de se placer sur l'échiquier de l'avant-garde littéraire en mobilisant un discours dénonciateur et en justifiant esthétiquement leur choix du thème de l'identité féminine. Cette volonté de réhabilitations 'exprime par une déclinaison du slogan féministe "mon corps m'appartient" [...]. Cette traduction littéraire des revendications féministes est érigé [sic] au rang de thématique subversive (Naudier, 2001: 64).

Esta actitud desafiante de subversión en la escritura remite sin duda a la idea de transgresión que comenta Lejeune en su artículo "L'Autobiographie et l'aveu sexuel"13. En cualquier caso, este tipo de escritura, "écriture-femme", que se desarrolla hacia mediados de los años 70 del siglo pasado, originará una confrontación con otras autoras -las llamadas "égalitaristes"- que rechazan la idea de una escritura de especificidad femenina: “'L'écriture femme', expression esthétique et théorique, [...] s'impose à partir de 1975 alors même que l'antagonisme avec l'autre tendance du mouvement des femmes, les 'égalitaristes', qui refusent l'idée d'une spécificité féminine, s'intensifie" (Naudier, 2001: 63). Sin llegar a hacer "escuela", la orientación estética de la temática del cuerpo femenino y la sexualidad, promovida por "l'écriture-femme", sigue presente en la producción de las autoras contemporáneas. Sin embargo, más que dependientes de un posicionamiento de grupo, la escritura femenina contemporánea se caracterizaría más bien por su carácter individualista, en lo que Naudier denomina "l'atomisation des positions" (2001: 71).

Se trata, en definitiva, para las mujeres de una manera de entrar a

página web del Centre dona i literatura, en la sección de Publicaciones (http://www.ub.edu/cdona/publicacions/ entrevistas-helene-cixous-no-escribim [8/09/2017]).

13. "L'aveu n'est, le plus souvent, ni un acte de confession (renoncement à la chose interdite), ni un acte de libération (dépassement définitif de l'interdit), mais un acte de transgression, en employant le mot dans le sens que lui donne G. Bataille. Il rend délicieusement sensible l'interdit, permet de le percevoir, d'en jouir" (Lejeune, 2008: 43). 
formar parte del canon literario ${ }^{14}$, de ahí que aquella postura subversiva de "l'écriture-femme", que hizo furor en la década de 1975-1985, viene a constituir una manifestación más de las "argucias" que ha desarrollado la mujer para acceder a un ámbito tradicionalmente de difícil acceso para ella, tal y como hace observar, entre otros, Montserrat Serrano, en su estudio "Quand écrire c'est se dire. De la vie à l'œuvre, la femme":

L'histoire nous permet de déceler quels ont été les chemins cachés suivis par les femmes pour prendre contact avec les voies d'accès à la lecture et à l'écriture; nous pouvons découvrir leurs ruses et leurs manœuvres pour pouvoir s'introduire dans le canon établi. C'est en cherchant à percer (discrètement) dans ce monde qu'elles ont défriché de nouveaux sentiers, des sentiers parfois tout simplement délaissés par les écrivains masculins: elles ont trouvé des champs littéraires particuliers pour développer leurs aptitudes [...]. Et ce long chemin aboutit à une richesse littéraire inconnue auparavant. Car nous sommes entrés au XXIe siècle, et le foisonnement d'écrivains femmes est certain (Serrano, 2004: 172).

La riqueza de la escritura femenina a la que alude Serrano se percibe de forma particular en la producción autobiográfica de nuestros días. Con todo, ya en el Antiguo Régimen encontramos numerosos ejemplos de memorias de mujeres, que han querido transmitir la relevancia histórica de sus testimonios: es el caso de Marguerite de Valois ${ }^{15}$, de Hortense y Marie Mancini, y en el marco de las guerras de La Fronda, de la Grande Mademoiselle, de Madame de Motteville, Marie d'Orléans o de Madame de la Guette. En su artículo "Les femmes, la Fronde et l'écriture de l'histoire", Sophie Vergnes analiza la participación de las "frondeuses",

14. Así como a facilitar su acceso en el campo editorial: "Prôner l'existence d'une écriture féminine s'inscrit dans une stratégie programmatique de la tendance 'différencialiste' qui a la possibilité de marquer sa présence dans le champ éditorial. Le discours sur la spécificité féminine est dès lors l'argument majeur permettant de justifier la création d'un espace exclusivement réservé aux femmes" (Naudier, 2001: 69).

15. Éliane Viennot, gran especialista en escritura femenina, se ha ocupado con profusión de la "Reine Margot" y ha reeditado recientemente sus memorias. Véase la página web de Viennot (http://www.elianeviennot. fr/Marguerite.html [15/07/2017]). 
así como el legado escrito de algunas de ellas: "les Mémoires de la Fronde contribuent à réintroduire les femmes dans l'histoire. Mieux, certaines de celles qui participèrent directement au conflit, ont laissé de leur expérience d'engagement des traces écrites"' (2011: 50).

Con la Revolución de 1789 y el advenimiento del Imperio surge una nueva razón para recoger la experiencia vital de tan marcados acontecimientos históricos, como harán Madame de Genlis, Madame de Campan, Madame Roland, Élisabeth Vigée Le Brun, Madame Chasteney o la duchesse d'Abrantès. Zanone estudia el fenómeno de la explosión de memorias publicadas en la primera mitad del siglo XIX en "Les mémorialistes et le pouvoir, ou comment l'aristocratie devint un thème littéraire", donde concluye: "Les Mémoires, qui étaient originellement le lieu d'un exercice écrit du pouvoir, lieu d'expression de son privilège exclusif, ont été aussi le lieu où se signe sa perte lisible dans l'écriture" (2000: 23).

La evolución progresiva de la producción autobiográfica en el XIX se manifiesta en autoras tan dispares como George Sand, Louise Michel o, ya a comienzos del XX, la comtesse de Martel. Pero será ciertamente en el siglo XX cuando la producción autobiográfica femenina adquiera todo su protagonismo, con autoras como Simone de Beauvoir, Nathalie Sarraute, Violette Leduc, Annie Ernaux, Chantal Chawaf, Chloé Delaume...

A partir del último tercio del siglo $\mathrm{XX}$ hasta nuestros días, la producción autobiográfica femenina se va a manifestar a través de una sinceridad absoluta, plagada de revelaciones íntimas:

La réserve observée par George Sand ou Simone de Beauvoir à l'égard de la confidence intime, notamment sexuelle, fait place, chez les jeunes écrivaines de ce début du XXIe siècle, à une sincérité totale. [...] Cet abandon des censures concerne également leurs aînées, qui, au même moment, lâchent aussi les amarres et passent de la liberté $d u$ 'faire' déjà conquise, à celle du 'dire'. Ainsi, après Passion simple (1991), qui cernait surtout les modifications comportementales déclenchées par l'obsession amoureuse, Annie Ernaux livre en 2001 aux lecteurs le journal intime tenu au moment où elle vivait cette passion (LecarmeTabone, 2010: 12). 
La exhibición de loíntimo, el destierro de toda inhibición, la revelación de las confesiones de carácter sexual, confluyen en autoras como Catherine Cusset o Christine Angot. Y es también aquí donde la autoficción adquiere protagonismo como recurso escritural por cuanto permite establecer una distancia, al tiempo que no sólo privilegia los "pactos ambiguos", sino también la fragmentación del relato. Lecarme-Tabone, en este contexto, considera que la renovación de la escritura autobiográfica por medio de los "récits de filiation" (que combinan biografía, autobiografía y novela) viene a probar una continuidad enriquecida de formas escriturales femeninas anteriores:

Mais n'est-ce pas là, plutôt, la continuation, sans doute enrichie, d'une forme déjà pratiquée par des femmes: Georges Sand (qui consacrait un tiers de son autobiographie à l'histoire de sa famille et plus particulièrement à la figure paternelle), Colette (qui ressuscitait et réinventait Sido), Marguerite Yourcenar (qui explorait sa double ascendance)? (2010: 19).

La preferencia por la autoficción tiene una incidencia particularmente significativa en el ámbito francófono, donde lo han desarrollado autoras como Amélie Nothomb, Suzanne Lilar, Nina Bouraoui, Assia Djebar, Leonora Miano... En este contexto, a partir de los estudios en la materia, Shirley Jordan hace observar que se trata de un rasgo distintivo de aquella escritura que busca la construcción de la identidad en zonas de tensión fuera de la Francia metropolitana: "Autofiction is seen to be linked to promoting individual and social change and to constructing identity in situations of tension and in locations outside metropolitan France" (2013: 82).

Por todo ello, concluiremos nuestro estudio reproduciendo la cita de Mounir Laouyen, elegida por Jordan para encabezar su artículo "Autofiction in the Feminine", por tratarse precisamente de una reflexión que pone de relieve la incidencia de la autoficción como forma autobiográfica femenina de preferencia en nuestros días: "L'autobiographie traditionnelle étant devenue impraticable, tout discours sur soi tend à devenir peu ou prou autofictionnel" (2013: 76). 


\section{ESTUDIOS DEL PRESENTE MONOGRÁFICO}

Una vez establecidos a grandes rasgos los fundamentos teóricos y críticos de la materia objeto de estudio en el presente monográfico, conviene recordar que todos los artículos que lo componen se desarrollan dentro de la perspectiva de un estado de la cuestión en el ámbito específico que aborda cada uno. En su elaboración han participado grandes especialistas en la materia que ofrecen en sus contribuciones un estudio minucioso, necesariamente acotado, de una parcela representativa del tema objeto de estudio, es decir, de la escritura autobiográfica femenina en lengua francesa, desde el siglo XIX a nuestros días.

Importa dejar constancia, a priori, de que el monográfico ni pretende ni puede ser exhaustivo, dada la amplitud de su ámbito de estudio, así como por el elevado número de autoras y de textos de carácter autobiográfico en lengua francesa, que hacen imposible su estudio completo en tan reducido espacio. Asimismo, hacemos observar la voluntad expresa de dar prioridad a la producción autobiográfica más reciente, así como la de abarcar el mundo francófono en nuestro campo de estudio, aún cuando, por idénticas razones, no se hayan podido incluir todas las francofonías.

Por lo demás, el monográfico aborda el estudio de la escritura autobiográfica femenina desde una doble perspectiva complementaria, razón por la que los artículos se han ordenado en dos partes, en función del enfoque acordado: en primer lugar, los que ofrecen un estudio panorámico más general, que puede aparecer ilustrado en una autora determinada, $y$, en un segundo momento, los que se centran exclusivamente en una sola autora.

Así, en la primera parte, el artículo de Mercè Boixareu (UNED), "Las memorias de principios del XIX y los Souvenirs de Élisabeth Vigée Le Brun", aborda la profusión de memorias publicadas en la primera mitad del siglo XIX, ilustradas en la obra de Vigée Le Brun. Le sigue el artículo de Elena Cuasante (Universidad de Cádiz), "Autobiogr-África", donde se analiza la escritura autobiográfica femenina francófona del África negra. Finalmente, el artículo de Marta Segarra (Universidad de Barcelona), "Vida y verdad: autobiografías de Hélène Cixous", aborda el fenómeno de la autoficción femenina y lo ilustra a través de la producción autobiográfica de la escritora de origen argelino Hélène Cixous (1937-), figura clave para entender los postulados de la "écriture-femme". 
Los estudios centrados en una autora determinada se ordenan siguiendo un criterio cronológico. Por ello, abre esta segunda parte el artículo de M. Carme Figuerola (Universitat de Lleida), que analiza los textos de autoficción de la escritora de origen ruso-ucraniano Élisabeth Gille (1937-1996) en "De Elisabeth Gille a Irène Némirovsky: recreación de un doble itinerario de vida a través de la escritura". A continuación, Francisca Romeral (Universidad de Cádiz), en su artículo intitulado "Annie Ernaux: una autobiografía sometida a constante autorrevisión", se centra en Ernaux (1940-) como figura de referencia dentro del panorama literario de la escritura autobiográfica femenina en Francia. Finalmente, cierra el monográfico el artículo de Montserrat Serrano (Universidad de Granada), dedicado a la escritora de origen argelino Nina Bouraoui (1967-) e intitulado "Nina Bouraoui: de Garçon manqué a Poupée Bella, itinerario vital de una consciencia".

Se trata, en definitiva, de un conjunto de estudios que permiten ilustrar la variedad y amplitud de las manifestaciones autobiográficas desarrolladas por autoras de distintos países y bagajes culturales, cuyo denominador común es el empleo del francés como lengua de expresión literaria.

\section{REFERENCIAS BIBLIOGRÁFICAS}

ALBERCA, M. (2007). El pacto ambiguo. De la novela autobiográfica a la autoficción. Madrid: Biblioteca Nueva.

BOIDARD BOISSON, C. (dir.) (2007). L'autobiographie dans l'espace francophone III: Le Maghreb. Cádiz: Servicio de Publicaciones de la Universidad de Cádiz. Colección Estudios de Francofonía.

BRUNEL, P. (2008). "Introduction aux autobiographies". Revue de littérature comparée 325, 7-22 (también en http://www.cairn.info/ revue-de-litterature-comparee-2008-1-page-7.htm [8/07/2017]).

BUENO ALONSO, J. (2004). "Femme, identité, écriture dans les textes francophones du Maghreb". Thélème. Revista Complutense de Estudios Franceses 19, 7-20.

BUSH, E. W. (1974). "L'autobiographie considérée comme acte littéraire". Poétique 17, 14-26. 
CAMARERO, J. (2008). "La théorie de l'autobiographie de Georges Gusdorf". Çédille 4, 57-82, https://cedille.webs.ull.es/cuatro/ camarero.pdf [04/08/2017].

CASAS, A. (2012). La autoficción: reflexiones teóricas. Madrid: Arco/ Libros.

COIRAULT, Y. (1975). "Autobiographie et mémoires (XVII'-XVIII" siècles) ou existence et naissance de l'autobiographie". Revue d'Histoire Littéraire de la France 6, 937-953.

COUDREUSE, A. (dir.) (2011). "Dossier: Les Mémoires, une question de genre?". Itinéraires 2011-1, http://itineraires.revues.org/1590 [09/08/2017].

DÍAZ NARBONA, I. (dir.) (2005). L'autobiographie dans l'espace francophone. II. L'Afrique. Cádiz: Servicio de Publicaciones de la Universidad de Cádiz. Colección Estudios de Francofonía.

DIDIER, B. (1981). L'écriture-femme. París: Presses Universitaires de France.

GANS-GUINOUNE, A.-M. (2009). "Autobiographie et francophonie: cache-cache entre 'nous' et 'je"'. Relief 3, 61-76, https://www.revuerelief.org/articles/abstract/10.18352/relief.410/ [09/08/2017].

GARCÍA, M. (2009). "L'étiquette générique autofiction: us et coutumes". Çédille 5, 146-163, http://webpages.ull.es/users/cedille/cinco/ garcia.pdf [04/08/2017].

GEHRMANN, S. \& GRONEMANN, C. (eds.) (2006). Les enJEux de l'autobiographique dans les littératures de langue française: du genre à l'espace, l'autobiographie postcoloniale, l'hybridité. París: L'Harmattan.

GRÈVE, M. de (2008). “L'autobiographie, genre littéraire?”. Revue de littérature comparée 325, 23-31 (también en http://www.cairn.info/ revue-de-litterature-comparee-2008-1-page-23.htm [8/07/2017]).

GUSDORF, G. (1975). "De l'autobiographie initiatique à l'autobiographie genre littéraire". Revue d'Histoire Littéraire de la France 6, 957994.

(1991). Les écritures du moi. Lignes de vie 1. París: Éditions Odile Jacob.

HART, F. R. (1970). "Notes for an Anatomy of Modern Autobiography". New Literary History I, 485-510.

HERSANT, M.; JEANNELLE, J.-L. \& ZANONE, D. (eds.) (2013). Le 
Sens du passé. Pour une nouvelle approche des Mémoires. Rennes: Presses Universitaires de Rennes.

HOWARD, W. L. (1974). "Some principles of Autobiography". New Literary History 2, 363-381.

HUBIER, S. (2005). Littératures intimes. Les expressions du moi, de l'autobiographie à l'autofiction. París: Armand Colin.

JEANNELLE, J.-L. (2008). Écrire ses mémoires au XXe siècle. Déclin et renouveau. París: NRF Éditions Gallimard. Bibliothèque des idées. (2011). "Le sexe des Mémoires". Itinéraires 2011-1, 13-28, http:// itineraires.revues.org/1596 [09/08/2017].

JORDAN, S. (2013). "Autofiction in the Feminine". French Studies 67, 7684 (también en https://academic.oup.com/fs/article/67/1/76/535584/ Autofiction-in-the-Feminine? keytype $=$ ref\&ijkey $=V 97 z c z G M 5 x s P d$ zt $[08 / 07 / 2017])$.

LECARME, J. \& LECARME-TABONE, É. (1999). L'autobiographie. París: Armand Colin.

LECARME-TABONE, É. (2010). "L'autobiographie des femmes". Fabula Littérature Histoire Théorie 7, Dossier: Y a-t-il une histoire littéraire des femmes, http://www.fabula.org/lht/7/lecarme-tabone. html [09/08/2017].

LEJEUNE, Ph. (1975a). Le Pacte autobiographique. París: Éditions du Seuil.

(1975b). "Autobiographie et histoire littéraire". Revue d'histoire littéraire 6, 903-930.

(2008). "L'Autobiographie et l'aveu sexuel". Revue de littérature comparée 325, 37-51 (también en http://www.cairn.info/revue-delitterature-comparee-2008-1-page-37.htm [08/07/2017]).

NAUDIER, D. (2001/1). "L'écriture-femme, une innovation esthétique emblématique". Sociétés contemporaines 44, $57-73$ (también en http://www.cairn.info/revue-societes-contemporaines-2001-4page-57.htm [08/07/2017]).

PLANTÉ, C. (2015) La petite sœur de Balzac: essai sur la femme auteur. Lyon: Presses Universitaires de Lyon (nouvelle édition révisée).

REGNIER, T. (2008). "De l'autobiographie à l'autofiction: une généalogie paradoxale". Revue de littérature comparée 325, 33-36 (también en http://www.cairn.info/revue-de-litterature-comparee-2008-1page-33.htm [10/07/2017]). 
REID, M. (2010). Des femmes en littérature. París: Belin, Colección L'Extrême contemporain.

RENOUPREZ, M. (2000). "L'Autobiographie en question: poétique d'un genre". En La Philologie Française à la croisée de l'an 2000: panorama linguistique et littéraire, M. Serrano Mañés; L. Avendaño Anguita \& M. del C. Molina Romero (coords.), vol. 2, 113-121. Granada: Ediciones Universidad de Granada.

RENOUPREZ, M. \& TORRE GIMÉNEZ, E. de la (dir.) (2003). L'autobiographie dans l'espace francophone I: La Belgique. Cádiz: Servicio de Publicaciones de la Universidad de Cádiz, Colección Estudios de Francofonía.

ROMERA CASTILlO, J. (2006). De primera mano. Sobre escritura autobiográfica en España (siglo XX). Madrid: Visor Libros.

(2010). "La escritura (auto)biográfica y el SELITEN@T: Guía bibliográfica”. Signa 19, 333-369 (también en http://descargas.cervantesvirtual.com/servlet/

SirveObras/12476280980181621332679/035521.pdf?incr $=1$ [20/07/2017]).

ROMERA CASTILLO, J. (ed.) (2003). Teatro y memoria en la segunda mitad del siglo XX. Madrid: Visor Libros.

ROMERA CASTILLO. J. \& GUTIÉRREZ CARBAJO, F. (eds.) (1998). Biografias literarias (1975-1997). Madrid: Visor Libros. (2000). Poesía histórica y (auto)biográfica (1975-1999). Madrid: Visor Libros.

ROMERA CASTILLO, J.; YLLERA, A.; GARCÍA-PAGE, M. \& CALVET, R. (eds.) (1993). Escritura autobiográfica. Madrid: Visor Libros.

RUBIALES BONILLA, L. (dir.) (2010). L'autobiographie dans l'espace francophone IV: Les Caraïbes et l'Océan Indien. Cádiz: Servicio de Publicaciones de la Universidad de Cádiz, Colección Estudios de Francofonía.

SEGARRA, M. (ed.) (2010). Entrevistas a Hélène Cixous. No escribimos sin cuerpo. Barcelona: Icaria.

SERRANO MAÑÉS, M. (2004). "Quand écrire c'est se dire. De la vie à l'œuvre, la femme". Thélème 19, 171-182 (también en https:// revistas.ucm.es/index.php/THEL/issue/view/THEL040411/showToc [15/07/2017]). 
VERGNES, S. (2011). "Des discours de la discorde: les femmes, la Fronde et l'écriture de 1'histoire". Études Épistémè 19, 50-65 (también en https://episteme.revues.org/627 [04/06/2017]).

ZANONE, D. (1990). "Les mémoires comme genre? La mémoire historique en quête d'une forme stable dans la première moitié du XIX ${ }^{\mathrm{e}}$ siècle français". Intercâmbio. Revue d'études françaises 1, 35-62 (también en http://ler.letras.up.pt/uploads/ficheiros/5942.pdf [03/06/2017]).

(2000). "Les mémorialistes et le pouvoir, ou comment l'aristocratie devint un thème littéraire". Lieux littéraires 2, 11-23 (también en https://dial.uclouvain.be/pr/boreal/en/object/boreal\%3A86318/ datastreams [03/06/2017]).

(2006). Écrire son temps. Les Mémoires en France de 1818 à 1848. Lyon: Presses Universitaires de Lyon.

Recibido el 27 de mayo de 2017.

Aceptado el 15 de septiembre de 2017. 
